\section{Conselho de saúde e efetividade participativa: estudo sobre avaliação de desempenho}

\author{
Health councils and participatory effectiveness: \\ a performance assessment study
}

\section{Consejo de salud y efectividad participativa: estudio sobre la evaluación de desempeño}

Maricélia Braga Rocha 1

Diane Costa Moreira 1

José Patrício Bispo Júnior 1

doi: 10.1590/0102-311X00241718

\section{Resumo}

O artigo tem por objetivo apresentar e analisar os resultados de um modelo de avaliação de desempenho de conselhos de saúde. O referencial teórico metodológico está fundamentado no método spidergram, adaptado à realidade dos conselhos de saúde. A matriz avaliativa considerou cinco dimensões de maior influência sobre a participação: autonomia, organização, representatividade, envolvimento comunitário e influência política. Com base na avaliação dos indicadores, foi estimado o valor de desempenho de cada dimensão e localizado no gráfico de cinco eixos. A aplicação da matriz foi realizada no Conselho de Saúde de Vitória da Conquista, Bahia, Brasil. Utilizou-se análise de documentos, observação das reuniões e entrevistas com 18 conselheiros como técnicas de coleta de dados. Os resultados demonstram nível avançado de autonomia do conselho com condições estruturais adequadas, porém, com limitações na independência financeira. A dimensão organização atingiu nível máximo de desempenho com a realização regular de reuniões, disponibilidade de informações para os conselheiros e funcionamento das comissões temáticas. A representatividade foi a dimensão de pior desempenho, demostrado pela frágil relação dos representantes com as entidades. A dimensão envolvimento comunitário apresentou nível avançado, com elevada participação de conselheiros e não conselheiros às reuniões e perfil de atuação propositivo. A dimensão influência politica obteve nível intermediário. Constatou-se elevada influência dos representantes sociais no processo deliberativo e diminuta capacidade de acompanhamento das políticas. A matriz utilizada mostrou-se adequada e viável para a avaliação de desempenho dos conselhos de saúde.

Participação Social; Conselhos de Saúde; Avaliação em Saúde; Deliberações; Política de Saúde

\author{
Correspondência \\ J. P. Bispo Júnior \\ Instituto Multidisciplinar em Saúde, Universidade \\ Federal da Bahia. \\ Rua Hormindo Barros 58, quadra 17, lote 58, Vitória da \\ Conquista, BA 45029-094, Brasil. \\ jpatricio@ufba.br \\ 1 Instituto Multidisciplinar em Saúde, Universidade Federal da \\ Bahia, Vitória da Conquista, Brasil.
}




\section{Introdução}

O envolvimento dos cidadãos nos processos deliberativos tem como propósito principal tornar as políticas públicas mais responsivas e adequadas às realidades das populações 1 . Parte-se do pressuposto de que a participação social tende a conduzir processos decisórios mais acertados 2, fortalece a promoção da saúde e o combate às desigualdades sociais ${ }^{3}$, além de possibilitar o desenvolvimento de mecanismos de controle mais efetivos ${ }^{4}$. Nesse contexto, o envolvimento público e a governança participativa têm assumido grande relevância nos sistemas de saúde dos países ocidentais 5 .

Em países europeus, as recentes reformas dos sistemas de saúde introduziram mecanismos participatórios, de diferentes formatos e dimensões, como elementos indutores da ampliação do impacto das ações de saúde 6. Também na América Latina, a participação foi constituída em estratégia prioritária para o alcance de resultados mais equitativos na saúde 7 e dos Objetivos de Desenvolvimento Sustentável 8 .

Embora a dimensão participativa seja elemento comum nas reformas dos sistemas sanitários, o sentido atribuído à participação varia consideravelmente entre os países. Serapioni et al. ${ }^{9}$ propõem a classificação dos mecanismos de envolvimento comunitário em três níveis: participação dos cidadãos; consulta pública; e comunicação pública. A participação dos cidadãos ocorre quando há a participação institucionalizada com legitimidade legal ou como participação ocasional, mas com o envolvimento dos usuários na priorização de necessidades, planejamento ou avaliação. A consulta pública está associada à promoção de atividades de escuta/consulta e não permite um diálogo formal entre os representantes do sistema de saúde e os usuários. E, por fim, a comunicação pública pode ser caracterizada por ter fluxo informativo unidirecional, isto é, das instituições e serviços de saúde para os cidadãos.

Nesse contexto, a participação social instituída no Sistema Único de Saúde (SUS) integra o nível mais elevado de acordo com a classificação anteriormente citada. Os conselhos de saúde são mecanismos institucionais, de caráter permanente, cuja metade dos membros é composta por representantes dos usuários e com função deliberativa 10. Não obstante os problemas ainda vivenciados, esse arranjo é considerado uma importante inovação democrática que possibilita a inserção da sociedade civil nos núcleos decisórios das políticas de saúde, estabelecendo novas relações entre Estado e sociedade 3.

Serapioni 11 ressalta a necessidade de fortalecimento dos mecanismos de participação social. Segundo o autor, a prioridade atual não é mais legitimar a participação nos sistemas de saúde, preocupação inerente às décadas de 1980 e 1990, mas sim garantir a qualidade e a efetividade da participação. Nesse contexto, a avaliação dos mecanismos de envolvimento comunitário se destaca como elemento fundamental para a promoção da efetividade participativa 12. Essa compreende a capacidade das instâncias de participação influenciarem, controlarem ou decidirem sobre o conteúdo, a abrangência e os rumos das políticas públicas 11,12. Envolve a institucionalização dos procedimentos, a pluralidade da composição, a deliberação inclusiva, o poder de agenda dos integrantes, o controle da decisão e o acompanhamento das ações públicas 13 .

Assim, duas dimensões são fundamentais para a avaliação da efetividade participativa. Uma delas diz respeito ao processo, em que se busca avaliar a qualidade das práticas de participação e os mecanismos de educação à democracia 11 . A outra está relacionada com os resultados da participação, cujo foco é identificar em que medida o envolvimento dos cidadãos consegue influenciar nas decisões e trazer benefícios para as comunidades 14 .

Vários autores apontam a carência de estudos avaliativos sobre as instâncias de participação dos cidadãos 11,14,15. Embora o envolvimento público seja defendido como meio para melhorar a capacidade de resposta dos sistemas de saúde, os benefícios esperados são justificados muito mais em pressupostos teóricos e normativos do que em resultados avaliativos consistentes 4,15,16.

A literatura nacional indica a existência de grandes entraves da participação por meio dos conselhos de saúde. Problemas relacionados à diminuta cultura cívica 17 , à representatividade 18 , ao clientelismo estatal 19, à baixa visibilidade dos conselhos 20 , à excessividade burocrática 21 e à manipulação de representantes sociais 22 estão entre os mais significativos. Ainda que tais estudos abordem importantes entraves e aspectos específicos da dinâmica dos conselhos de saúde, observa-se também no cenário nacional a carência de estudos avaliativos sobre a efetividade participativa.

Frente a esse contexto, o presente artigo tem por objetivo apresentar e analisar os resultados de um modelo de avaliação de desempenho de conselhos de saúde. 


\section{Aspectos teórico-metodológicos}

Pesquisa avaliativa centrada nos aspectos de desempenho das instâncias de participação social. O referencial teórico metodológico está fundamentado no método spidergram, desenvolvido por Rifkin et al. 23 .

O método compreende a avaliação de cinco dimensões de maior influência sobre a participação. No método de Rifikin et al. 23, são usadas as seguintes dimensões: avaliação das necessidades (needs assessment); liderança (leadership); organização (organization); mobilização de recursos (resource mobilization); e gestão (management). Com base na sistematização dessas dimensões e da avaliação dos seus respectivos indicadores é possível medir, localizar e representar visualmente os níveis de desempenho da participação em saúde 24 . O spidergram tem sido utilizado em diversas experiências internacionais para avaliar a efetividade das instâncias de participação em diversos contextos 24,25,26,27.

Para o presente estudo, foram desenvolvidas adaptações do método ao contexto brasileiro e ao formato da participação institucionalizada no âmbito do SUS. A matriz de avaliação considerou os aspectos estruturais, organizativos e finalísticos dos conselhos de saúde 10. As dimensões foram definidas e sistematizadas baseando-se na revisão da literatura especializada que analisa as características, o funcionamento e os desafios dos conselhos de saúde. Dessa forma, a matriz foi composta pelas seguintes dimensões: autonomia; organização; representatividade; envolvimento comunitário; e influência política.

A dimensão autonomia avalia o nível de independência do conselho em relação à gestão da saúde, relaciona-se também às condições de funcionamento do conselho de saúde. Essa dimensão utilizou os indicadores: estrutura física (sede própria, linha telefônica e computador com acesso à Internet); secretaria executiva ou equivalente; orçamento próprio; e eleição para a mesa diretora. Os indicadores foram classificados como presentes ou ausentes.

A dimensão organização enfoca a dinâmica cotidiana do conselho. Relaciona-se à periodicidade e organização do plenário, assim como a existência e funcionamento de comissões temáticas. Leva em consideração dois indicadores: organização do plenário e organização das comissões. Para o plenário, foram consideradas as variáveis frequência das reuniões (ampla, moderada, restrita ou insuficiente) e encaminhamento prévio da pauta e material de apoio (presente ou ausente). $\mathrm{O}$ indicador comissões considera as variáveis existência de comissões (presente ou ausente) e periodicidade das reuniões (ampla, avançada, moderada ou insuficiente).

Por sua vez, a representatividade tem por interesse a relação estabelecida entre os representantes e os representados, com destaque para a interação e acompanhamento dos representantes por parte dos representados e os tipos de interesses representados pelos conselheiros. Essa dimensão considera os indicadores: composição do plenário (paritário ou não paritário); escolha dos representantes pelas entidades (eleitoral ou não eleitoral); relação regular entre representante e entidade/representados (forte ou frágil); e tipo de interesse representado (ampliado, corporativo ou individual).

A dimensão envolvimento comunitário considera o nível de engajamento e de participação das representações sociais no âmbito do conselho, sejam os conselheiros ou o público em geral. Para tanto, foram utilizados os indicadores: frequência dos conselheiros representantes sociais às reuniões (ampla, avançada, restrita ou insuficiente); frequência de não conselheiros (elevada, regular ou baixa); fala de não conselheiros nas reuniões (frequente, intermediária ou baixa); e perfil de atuação dos representantes sociais (avaliativo/propositivo, reivindicativo ou votante).

Por fim, a dimensão influência política contempla as relações de poder nos espaços interno (relações estabelecidas entre os conselheiros) e externo (capacidade em acompanhar a implantação das políticas de saúde) do conselho. Os indicadores foram: poder de agenda dos representantes sociais (amplo, moderado, restrito ou insuficiente); conteúdo dos temas pautados e deliberados (prestação de contas e aprovação de relatórios; participação social; organização e funcionamento dos serviços de saúde; estado de saúde da população e políticas públicas abrangentes) e acompanhamento da implantação das políticas (elevado, intermediário ou baixo).

Para cada dimensão, foi desenvolvido um continuum com a localização do nível de desempenho em uma escala de 1 a 5, em que o valor 1 corresponde ao nível incipiente e o valor 5 indica amplo desenvolvimento da dimensão. No Quadro 1 são apresentados os cinco níveis usados e os fatores considerados para a definição de cada nível. 


\section{Quadro 1}

Níveis e características das dimensões avaliativas dos conselhos de saúde.

\begin{tabular}{|c|c|c|c|}
\hline DIMENSÃO & INDICADORES & VARIÁVEL & RESULTADO \\
\hline \multirow{7}{*}{ Autonomia } & \multirow{4}{*}{ Estrutura física } & Sede própria & Sim \\
\hline & & Linha telefônica & Sim \\
\hline & & Computador & Sim \\
\hline & & Acesso à Internet & Sim \\
\hline & Apoio técnico & Secretaria executiva ou equivalente & Sim \\
\hline & Recursos financeiros & Orçamento próprio & Não \\
\hline & Coordenação do conselho & Eleição para presidência/mesa diretora & Sim \\
\hline \multirow{5}{*}{ Organização } & \multirow{3}{*}{ Plenário } & Periodicidade das reuniões plenárias & Ampla \\
\hline & & Encaminhamento prévio de pauta & Sim \\
\hline & & $\begin{array}{l}\text { Encaminhamento prévio de } \\
\text { material de apoio }\end{array}$ & Sim \\
\hline & \multirow{2}{*}{ Comissões } & Existência de comissões & Sim \\
\hline & & Periodicidade de reuniões das comissões & Moderada \\
\hline \multirow{4}{*}{ Representatividade } & Composição do conselho & Paridade do plenário & Adequada \\
\hline & Escolha dos representantes & $\begin{array}{l}\text { Mecanismo de escolha } \\
\text { dos representantes }\end{array}$ & Não eleitoral \\
\hline & $\begin{array}{c}\text { Relação entre representante e entidade/ } \\
\text { representados }\end{array}$ & $\begin{array}{l}\text { Discussão de temas com } \\
\text { diretoria da entidade }\end{array}$ & Frágil \\
\hline & Representação de interesses & Tipo de interesse representado & Ampliado \\
\hline \multirow{4}{*}{$\begin{array}{l}\text { Envolvimento } \\
\text { comunitário }\end{array}$} & Presença de conselheiros & Frequência de comselheiros às reuniões & Ampla \\
\hline & Presença de não conselheiros & $\begin{array}{l}\text { Frequência de não conselheiros } \\
\text { às reuniões }\end{array}$ & Elevada \\
\hline & Participação de não conselheiros & Fala de não conselheiros nas reuniões & Frequente \\
\hline & Prática participativa de conselheiros & $\begin{array}{l}\text { Perfil de atuação dos } \\
\text { representantes sociais }\end{array}$ & Avaliativo/Propositivo \\
\hline \multirow{4}{*}{ Influência política } & Poder de agenda & $\begin{array}{l}\text { Possibilidade de inserção de temas } \\
\text { pelos representantes sociais }\end{array}$ & Amplo \\
\hline & Temas pautados & Conteúdo dos temas pautados & - \\
\hline & Conteúdo das deliberações & Tipo de deliberação & - \\
\hline & Homologação e cumprimento das deliberações & $\begin{array}{l}\text { Homologação e cumprimento } \\
\text { das deliberações }\end{array}$ & Intermediário \\
\hline
\end{tabular}

O spidergram consiste na expressão gráfica do nível de desenvolvimento das dimensões avaliadas e da relação entre elas. Trata-se de um gráfico de cinco eixos, que faz referência a uma teia de aranha. Em cada eixo é marcado o nível identificado da dimensão e posteriormente ligado aos outros quatro. $\mathrm{Na}$ análise do gráfico, cada eixo é utilizado para demonstrar o grau de amplitude da dimensão. Com a ligação dos eixos, é possível visualizar o nível de participação da instância avaliada e as dimensões que, eventualmente, têm níveis mais avançados ou frágeis de desenvolvimento. Os formatos mais centrífugos representam estados avançados de participação, ao passo que uma configuração mais próxima do centro denota nível restrito de desenvolvimento.

O estudo foi conduzido no Município de Vitória da Conquista, Bahia. Essa é a terceira maior cidade do estado, com população de 338.885 habitantes 28. Conta com bom nível de organização dos serviços de saúde e forte tradição associativa 29. O Conselho Municipal de Saúde (CMS) foi criado em 1994 e é composto por 24 membros titulares. 
Os dados foram obtidos por meio de observação direta, análise documental e entrevistas semiestruturadas. Foram observadas reuniões plenárias do conselho e das comissões temáticas no período de setembro de 2017 a junho de 2018. Os documentos analisados foram: atas das plenárias de 2016 e 2017, regimento interno, listas de frequência das reuniões e deliberações do conselhos de saúde e atos normativos da Secretaria Municipal de Saúde. Foram entrevistados 18 conselheiros, entre abril e junho de 2018, escolhidos pelo protagonismo ou articulação observado nas reuniões, contemplando todos os segmentos representados.

O roteiro das entrevistas foi composto por questões gerais sobre a organização e funcionamento do conselho de saúde e por questões específicas sobre cada uma das dimensões. Assim, foram abordados aspectos relacionados à estrutura e coordenação do conselho, participação em comissões temáticas, relações estabelecidas com os representados, critérios para a formação de posicionamentos e formas de acompanhamento das deliberações proferidas. Os dados e informações foram categorizados com o uso da técnica de análise de conteúdo temática proposta por Gibbs 30.

A realização do estudo foi aprovada pela plenária do CMS de Vitória da Conquista. Também foi aprovado pelo Comitê de Ética em Pesquisa do Instituto Multidisciplinar em Saúde da Universidade Federal da Bahia, parecer no 2.485.076. Todos os participantes assinaram Termo de Consentimento Livre e Esclarecido.

\section{Resultados}

Os resultados demonstraram variação no desempenho das dimensões analisadas. O spidergram (Figura 1) ilustra os valores obtidos em cada dimensão e possibilita visualizar aquelas de melhor desempenho e as que apresentam maiores fragilidades. No Quadro 2, estão sistematizados os resultados dos indicadores que compõem cada dimensão.

No que se refere à autonomia, o conselho apresentou nível avançado, com escore 4 (Figura 1). Os resultados revelaram que dos quatro indicadores dessa dimensão, três são plenamente contemplados. O conselho tem estrutura física adequada com sede própria, linha telefônica e computador com acesso à Internet (Quadro 2). Quanto ao apoio técnico, o conselho dispõe de estrutura equivalente à de secretaria executiva, com uma coordenadora de controle social e duas servidoras de apoio administrativo.

A inexistência de dotação orçamentária própria mostrou interferir na autonomia do conselho. Para o desenvolvimento das atividades do CMS, o suporte financeiro é viabilizado pelo gabinete do secretário de saúde, o que foi relacionado ao comprometimento da autonomia. "As ações do conselho, quando precisam ser executadas, tem que fazer um ofício, mandar para a secretária e ela delibera. A depender da disponibilidade, eles liberam ou não" (Entrevistado 13 - Prestador).

A coordenação do conselho se mostrou independente da gestão municipal. São realizadas eleições bianuais para a mesa diretora composta por presidente, vice-presidente e secretária. A atuação da mesa diretora foi destacada pelos entrevistados por apresentar postura independente e democrática, o que foi associado como elemento de fortalecimento da autonomia. "O nosso conselho que não tem o secretário de saúde como presidente, é um ganho importantíssimo pra a gente. Eu acho que é fundamental" (Entrevistado 15 - Trabalhador).

A organização do conselho obteve escore máximo do spidergram (Figura 1). Conforme observado no Quadro 2, todos os indicadores dessa dimensão foram plenamente contemplados. O conselho mantém calendário regular e todas as reuniões previstas para o período foram realizadas. Além disso, o próprio conselho demandou a necessidade de reuniões extraordinárias para a discussão de temas complexos e que necessitavam de maior aprofundamento. A disponibilidade prévia dos balancetes contábeis e de outros documentos relativos às prestações de contas favoreceu a maior capacidade de controle das finanças por parte dos conselheiros.

Em relação às comissões temáticas, foi identificada a existência de cinco comissões permanentes: Comissão de Orçamento e Finanças; Comissão de Ética; Comissão Intersetorial de Mobilização Popular; Comissão Intersetorial de Saúde do Trabalhador; e Comissão de Saúde. A lógica de funcionamento variou de acordo com cada comissão. Algumas atuam a partir da demanda do plenário, a exemplo da Comissão de Saúde, e outras têm a atividade regular, como a Comissão de Orçamento e 
Quadro 2

Desempenho das variáveis e indicadores, segundo dimensões avaliadas. Conselho Municipal de Saúde de Vitória da Conquista, Bahia, Brasil, 2018.

\begin{tabular}{|c|c|c|c|}
\hline DIMENSÃO & INDICADORES & VARIÁVEL & RESULTADO \\
\hline \multirow{7}{*}{ Autonomia } & \multirow{4}{*}{ Estrutura física } & Sede própria & Sim \\
\hline & & Linha telefônica & Sim \\
\hline & & Computador & Sim \\
\hline & & Acesso à Internet & Sim \\
\hline & Apoio técnico & Secretaria executiva ou equivalente & Sim \\
\hline & Recursos financeiros & Orçamento próprio & Não \\
\hline & Coordenação do conselho & Eleição para presidência/mesa diretora & Sim \\
\hline \multirow{5}{*}{ Organização } & \multirow{3}{*}{ Plenário } & Periodicidade das reuniões plenárias & Ampla \\
\hline & & Encaminhamento prévio de pauta & Sim \\
\hline & & $\begin{array}{l}\text { Encaminhamento prévio de } \\
\text { material de apoio }\end{array}$ & Sim \\
\hline & \multirow{2}{*}{ Comissões } & Existência de comissões & Sim \\
\hline & & Periodicidade de reuniões das comissões & Moderada \\
\hline \multirow{4}{*}{ Representatividade } & Composição do conselho & Paridade do plenário & Adequada \\
\hline & Escolha dos representantes & $\begin{array}{l}\text { Mecanismo de escolha dos } \\
\text { representantes }\end{array}$ & Não eleitoral \\
\hline & $\begin{array}{c}\text { Relação entre representante e entidade/ } \\
\text { representados }\end{array}$ & $\begin{array}{l}\text { Discussão de temas com diretoria } \\
\text { da entidade }\end{array}$ & Frágil \\
\hline & Representação de interesses & Tipo de interesse representado & Ampliado \\
\hline \multirow{4}{*}{$\begin{array}{l}\text { Envolvimento } \\
\text { comunitário }\end{array}$} & Presença de conselheiros & Frequência de comselheiros às reuniões & Ampla \\
\hline & Presença de não conselheiros & $\begin{array}{l}\text { Frequência de não conselheiros } \\
\text { às reuniões }\end{array}$ & Elevada \\
\hline & Participação de não conselheiros & Fala de não conselheiros nas reuniões & Frequente \\
\hline & Prática participativa de conselheiros & $\begin{array}{l}\text { Perfil de atuação dos } \\
\text { representantes sociais }\end{array}$ & Avaliativo/Propositivo \\
\hline \multirow{4}{*}{ Influência política } & Poder de agenda & $\begin{array}{c}\text { Possibilidade de inserção de temas pelos } \\
\text { representantes sociais }\end{array}$ & Amplo \\
\hline & Temas pautados & Conteúdo dos temas pautados & - \\
\hline & Conteúdo das deliberações & Tipo de deliberação & - \\
\hline & Homologação e cumprimento das deliberações & $\begin{array}{l}\text { Homologação e cumprimento } \\
\text { das deliberações }\end{array}$ & Intermediário \\
\hline
\end{tabular}

Finanças. As comissões de Orçamento e Finanças e a Intersetorial de Saúde do Trabalhador demonstraram maiores níveis de atividade.

A dimensão representatividade obteve o valor mais centrípeto do spidergram, com escore 2 (Figura 1). Dos quatro indicadores dessa dimensão, apenas dois apresentaram resultados de desempenho elevado (Quadro 2). Constatou-se que o conselho tem formação paritária e composição que privilegia a representação de segmentos sociais e populares. Na distribuição dos assentos pela natureza da representação - governamentais, de mercado ou representantes sociais - a configuração mostrou predomínio de representantes sociais. Identificou-se que $75 \%$ dos assentos (18 das 24 vagas) são de representações populares ou de trabalhadores. A representação de mercado corresponde a apenas uma das 24 vagas.

Os indicadores escolha dos representantes e relação representante-entidade apresentaram frágil desempenho. O predomínio de critérios não eleitorais para a escolha dos representantes foi relacionado ao desinteresse dos integrantes das entidades. "Foi um consenso [da diretoria]. As outras pessoas 
Figura 1

Nível de participação social, segundo dimensões. Conselho Municipal de Saúde de Vitória da Conquista, Bahia, Brasil, 2018.

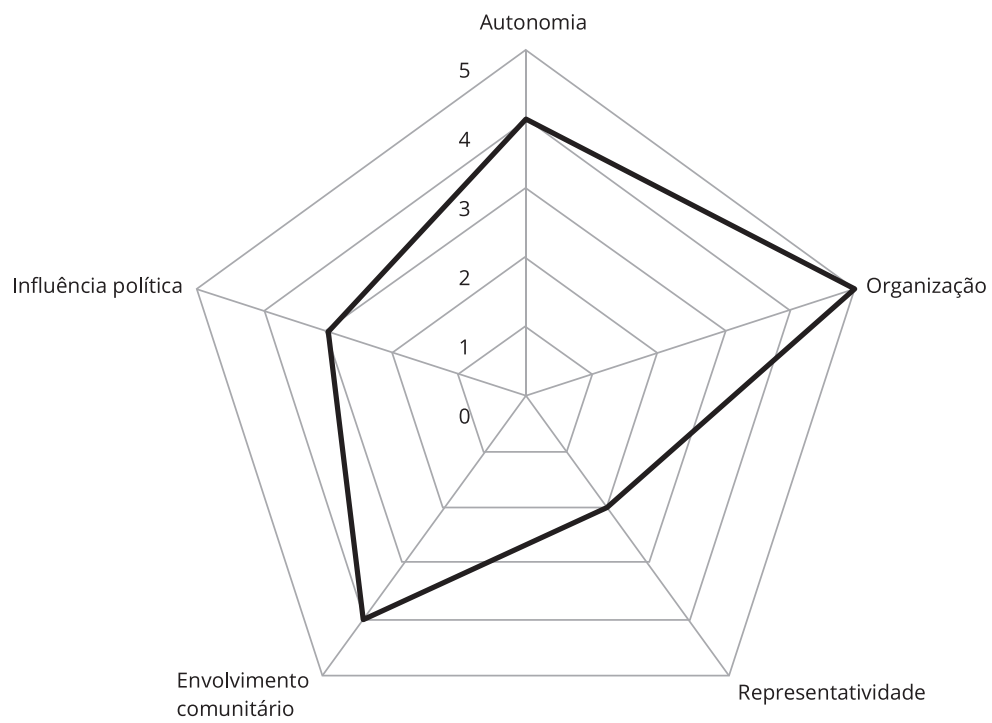

não estavam interessadas e eu estava, porque eu achava que tinha que ter uma representação" (Entrevistado 6 - Usuário).

Não é prática comum os conselheiros discutirem com os representados as temáticas tratadas no CMS. Apenas as representações de natureza coorporativa e de defesa de interesses específicos, a exemplo dos profissionais de saúde, mantiveram contato frequente com os segmentos representados. "Quando essa pauta chegava, a gente já fazia reuniões para discutir como seria a condução nas reuniões, sempre eu ficava muito preso àquilo que era da nossa responsabilidade” (Entrevistado 2 - Trabalhador).

Mesmo com os interesses corporativos de alguns membros, os resultados evidenciaram a predominância da representação de interesses do tipo ampliado. Nesse sentido, a postura dos conselheiros esteve direcionada à defesa do SUS e às políticas abrangentes de saúde, o que foi relacionado ao bom desempenho do conselho.

Sobre o envolvimento comunitário, a Figura 1 demonstra desempenho avançado, com escore 4. $\mathrm{Na}$ avaliação do indicador frequência dos conselheiros, verificou-se elevada assiduidade às plenárias. O percentual geral de frequência foi de 75\%. Quando estratifica-se por segmento, observam-se consideráveis diferenças entre os tipos de representação. Os representantes da gestão com $88 \%$ de frequência; os prestadores com 73\%; os profissionais de saúde apresentaram 75\%; e os usuários $63 \%$.

Quanto à frequência e participação de não conselheiros às plenárias, identificou-se números significativos de comparecimento e intervenções. No entanto, também foi observado que a participação desse público ocorre por grupos de interesses específicos, a exemplo de integrantes da gestão e estudantes de Ensino Superior. Verificou-se baixa participação de usuários de segmentos populares.

Sobre o perfil de atuação, foi evidenciado que os representantes sociais desempenham atuação predominantemente do tipo avaliativo/propositivo (Quadro 2), o que foi relacionado à capacidade do conselho em discutir temas abrangentes sobre saúde. A avaliação permitiu também identificar que a prática participativa vai além daquela desenvolvida em plenário. Foi evidenciado um perfil de atuação articulada dos representantes sociais. Alguns dos representantes não se expressam com frequência no plenário, no entanto, estes se articulam, ouvem e opinam de forma paralela às reuniões. 
Na dimensão influência política, o conselho obteve escore mediano, sinalizando nível de desenvolvimento intermediário (Figura 1). A avaliação dos indicadores evidenciou elevado poder de agenda dos representantes sociais, burocratização dos temas discutidos e das deliberações, e frágil capacidade de acompanhamento do cumprimento das deliberações.

A Figura 2 mostra os porcentuais dos conteúdos discutidos e deliberados por grupos de temas. Sobre os assuntos pautados, observa-se que a maior parte do tempo do conselho foi direcionada às prestações de contas e discussões sobre o próprio funcionamento do conselho. De maneira semelhante, os temas deliberados estão concentrados sobre a participação e controle social e os processos de prestação de contas. A organização dos serviços e o desenvolvimento de políticas públicas corresponderam a proporções significativamente menores.

Por fim, constatou-se não existir rotina para a homologação das deliberações por parte do gestor. Os resultados demonstraram que parte das deliberações não foi homologada ou executada. Mesmo após discussões acaloradas e disputas acirradas de determinados temas, o conselho pouco se ocupou da fiscalização e acompanhamento das ações e políticas sobre as quais deliberou.

\section{Discussão}

A aplicação da matriz avaliativa corroborou algumas dificuldades sinalizadas na literatura e desvelou características pouco abordadas nos estudos nacionais. Das cinco dimensões analisadas, três apresentaram escores equivalentes ao nível elevado ou amplo, uma dimensão apresentou nível moderado e a representatividade demostrou nível restrito de desempenho. Assim, pode-se inferir que o conselho em estudo tem bom nível de desempenho, embora alguns indicadores sinalizem fragilidades consistentes.

No que se refere à autonomia, o conselho demonstrou ter uma elevada capacidade para o desempenho das atividades. A boa estrutura física e a presença de apoio técnico constituem importantes condições que favorecem o desenvolvimento das ações. Em um estudo censitário sobre os conselhos de saúde no Brasil, Moreira \& Escorel 31 revelam que ter sede própria e secretaria executiva não é realidade para mais de $2 / 3$ dos conselhos do país.

\section{Figura 2}

Proporção das pautas e das deliberações, por grupos de temas. Conselho Municipal de Saúde de Vitória da Conquista, Bahia, Brasil, 2018.

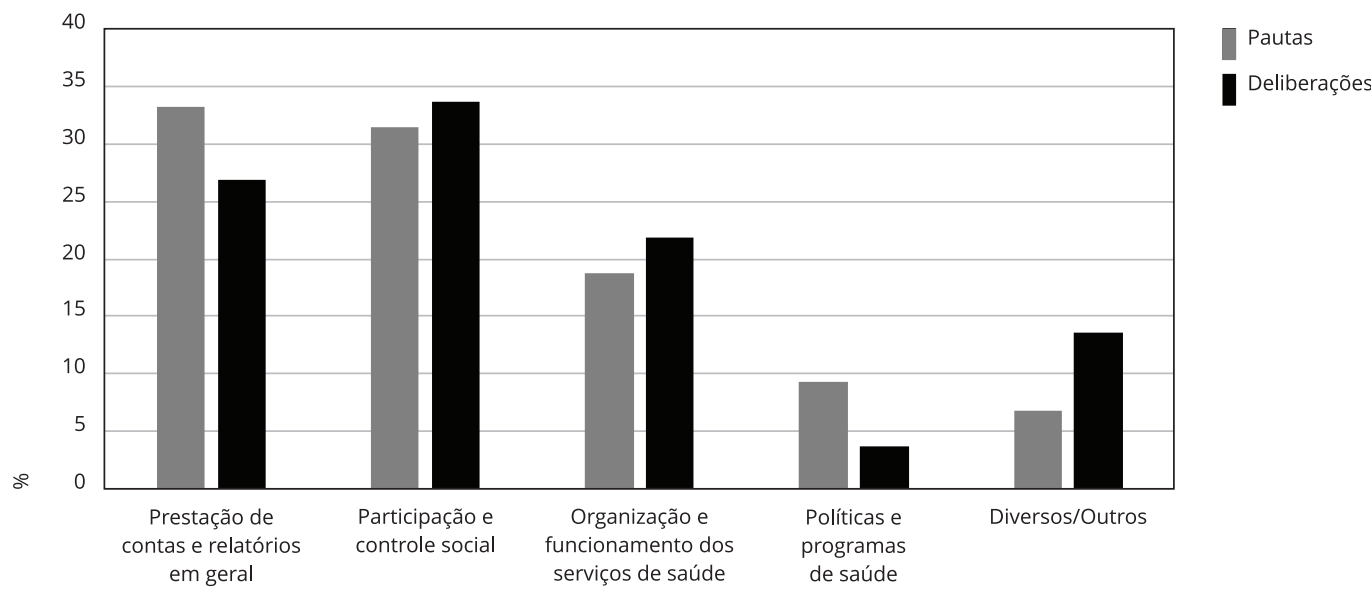


A realização de eleição para a mesa diretora viabilizou dissociar a função de presidente do conselho do segmento da gestão. Assim, tal prática potencializa a autonomia, pois possibilita postura democrática e independente na condução dos trabalhos. Nesse aspecto, os achados demonstraram relevante avanço quando comparados com a realidade descrita por Fuks 32 . O autor identificou que em Curitiba (Paraná) a presidência do CMS é ocupada de forma permanente pelo gestor municipal da saúde.

Por sua vez, o CMS de Vitória da Conquista sinalizou que a ausência de orçamento próprio constitui um fator limitante da autonomia. Apesar de ter sido identificado o suprimento de recursos para viabilizar as atividades do plenário, a independência do conselho mostrou-se comprometida, pois a disponibilidade dos recursos financeiros está atrelada ao julgamento e autorização do gestor municipal. A limitação da autonomia financeira é uma realidade comum aos conselhos de saúde 31 . Vieira \& Calvo 33 identificaram que a inexistência de orçamento próprio é uma fragilidade também dos conselhos de saúde dos municípios de Santa Catarina.

A dimensão organização foi a única que apresentou nível amplo de desenvolvimento. A regularidade das reuniões indica um nível de organização superior quando comparado com outras localidades 21,34 que apresentam dificuldades de quórum para se reunir. O encaminhamento prévio de pautas e material de apoio sinaliza boa organização e favorecem a accountability. De acordo com Siponi \& Silva 35 , a disponibilidade prévia de informações e materiais é importante para qualificar o debate e propiciar o amadurecimento do próprio conselheiro sobre a ideia a ser discutida no plenário. Também a existência das comissões constitui avanço ao desenvolvimento do conselho, pois possibilita que assuntos específicos sejam avaliados de maneira aprofundada.

Embora o elevado grau de organização seja um aspecto positivo e esperado dos conselhos, essa dimensão não deve ser analisada acriticamente. Shimizu et al. 36 destacam que os conselhos têm avançado nos níveis de organização formal para a operacionalização das atividades cotidianas. Dessa forma, as próprias gestões municipais buscam garantir os encontros mensais por receio de contingenciamento de recursos por parte das esferas estadual e federal. Nesse sentido, Durán \& Gerschman 19 refletem que os conselhos de saúde assumiram postura de elevada institucionalização, focados em uma linha de trabalho que, em última instância, hierarquiza-se em função das demandas burocráticas.

A representatividade foi a dimensão que obteve o pior resultado no cômputo geral, embora tenha apresentado nível avançado no indicador representação de interesses. A fragilidade nas relações com as entidades e as debilidades no processo de escolha dos representantes são elementos que comprometem a representatividade. Essa problemática mostrou-se também presente em outros conselhos 17. A distância entre representante e representados e as dificuldades de prestação de contas para as bases representadas revelam um processo de fragilização da capacidade de organização da sociedade civil 35 .

Cabe refletir que a representação no âmbito dos conselhos difere consideravelmente do tipo de representação eleitoral clássica. É um tipo de representação que favorece a democracia participativa em que a atuação do representante é sempre mais forte quando está aliada ao envolvimento dos representados. Assim, a participação nos conselhos gestores deve sempre buscar confluir características da representação institucional com mecanismos de mobilização social 18.

Os resultados correspondentes à dimensão envolvimento comunitário demonstram desempenho avançado. A elevada assiduidade dos conselheiros às reuniões corroborou a frequência regular das reuniões plenárias. Os indicadores frequência e fala de não conselheiros identificaram números significativos de comparecimento e intervenções. Todavia, isso não pode ser associada a um amplo envolvimento da comunidade, pois a participação esteve restrita a grupos específicos. Embora as plenárias sempre contassem com numerosa assistência, a realidade observada foi de limitado envolvimento da sociedade civil.

Destaca-se nessa dimensão a predominância de postura avaliativa/propositiva dos representantes sociais, o que constitui importante elemento para o bom desempenho da instância participativa. Nesse caso, o perfil propositivo do conselho pode ser resultante do elevado grau de articulação e de cooperação entre usuários e profissionais. Para Coelho 37 , a interface entre os representantes sociais, cada um com sua singularidade e importância, favorece a integração de saberes e forças dentro do conselho. Nesse sentido, diversos autores ratificam o pensamento que a forte implicação da sociedade civil é pré-condição para um desenho participativo exitoso 3,4,19,20,38. 
Quanto à influência política, o nível intermediário de desempenho sinaliza uma disparidade em relação aos dois aspectos centrais desta dimensão: o processo participativo e os resultados da participação. Os conselheiros demonstraram elevado poder de agenda, o que está relacionado positivamente com a qualidade do processo participativo. A capacidade de representantes sociais induzirem discussões de grande interesse social é reveladora da proatividade do conselho e evidencia o elevado grau de independência e compromisso com a coletividade. Diferentemente de outras realidades 36, o CMS de Vitória da Conquista demostrou não estar submetido à vontade do poder executivo local.

Por sua vez, o indicador relacionado aos resultados do processo participativo demonstrou dificuldades operacionais para ser viabilizado. Isso se expressou na incipiente capacidade de monitoramento da execução da política. Para além da realidade estudada, acompanhar a implantação das deliberações é algo complexo e cheio de incertezas 11. A institucionalização da participação pressupõe a possibilidade dos envolvidos exercerem algum grau de influência sobre os resultados da política 1 . No entanto, a experiência internacional demonstra que existem grandes dificuldades para se estimar a efetividade da participação em torno de benefícios de saúde para a população em geral 15,39.

Na realidade dos conselhos de saúde, tal perspectiva se mostra igualmente complexa. O caráter deliberativo pressupõe que as decisões sobre as políticas de saúde perpassem por definições do plenário ${ }^{3}$. No entanto, conforme destacam Wendhausen \& Cardoso 40 , o processo decisório é composto por uma série de decisões tomadas em diferentes instâncias e em diferentes momentos. Nesse sentido, dificilmente uma deliberação do conselho se converterá em política da maneira literal como foi aprovada. No processo de implantação é natural a necessidade de adequações técnicas, de arranjos para acomodar pressões de grupos de interesses e adaptações relacionadas à disponibilidade dos recursos.

\section{Considerações finais}

A avaliação empreendida demonstrou, de maneira geral, um nível amplo de participação no conselho. No entanto, chama a atenção a discrepância nos valores entre as dimensões. Enquanto a organização apresentou nível amplo de desempenho, e a autonomia e o envolvimento comunitário nível avançado, a dimensão representatividade expressou desenvolvimento restrito. Nesse sentido, o modelo multidimensional mostrou-se oportuno para identificar as potencialidades a serem valorizadas e as fragilidades que demandam maior atenção.

Com base nos achados, é sugestivo o desenvolvimento de algumas estratégias com o propósito de superar as fragilidades demonstradas pelos indicadores: efetivação da autonomia financeira, por meio da dotação orçamentária própria; incentivo ao fortalecimento das entidades de representação social e a maior interface destas com os representantes; promoção de ações para maior visibilidade do conselhos de saúde; criação de rotina para homologação e divulgação das deliberações.

Por fim, cabe refletir sobre o uso do método spidergram para a avaliação de desempenho dos conselhos de saúde. As adaptações realizadas e as dimensões sintetizadas mostraram-se adequadas às características da participação institucionalizada no Brasil. Também a expressão gráfica do spidergram é uma ferramenta oportuna, de fácil entendimento e adequada para sumarizar conjuntamente os níveis de desempenho de cada uma das cinco dimensões.

Como limitação do estudo, pode-se destacar que a ferramenta foi aplicada em um município de grande porte e com forte tradição participativa. A fim de aprimoramento do método, sugere-se que ele possa ser testado em outras localidades, especialmente em municípios de médio e pequeno portes. Nesse contexto, os resultados da aplicação em municípios menores podem revelar diferentes problemáticas e diferentes fragilidades que interferem no desempenho dos conselhos. 


\section{Colaboradores}

M. B. Rocha contribuiu na coleta, análise e interpretação dos dados, redação do manuscrito e aprovação da versão final a ser publicada. D. C. Moreira contribuiu na análise e interpretação dos dados, redação do manuscrito e aprovação da versão final. J. P. Bispo Júnior contribuiu na concepção do estudo, planejamento, análise e interpretação dos dados, redação do manuscrito e aprovação da versão final.

\section{Informações adicionais}

ORCID: Maricélia Braga Rocha (0000-0002-79829211); Diane Costa Moreira (0000-0003-12637677); José Patrício Bispo Júnior (0000-0003-41559612).

\section{Referências}

1. Degeling C, Carter SM, Rychetnik L. Which public and why deliberate? A scoping review of public deliberation in public health and health policy research. Soc Sci Med 2015; 131:114-21.

2. Coelho VSP. A democratização dos conselhos de saúde: o paradoxo de atrair não aliados. Novos Estudos Cebrap 2007; (78):77-92.

3. Bispo Júnior JP, Gerschman S. Potencial participativo e função deliberativa: um debate sobre a ampliação da democracia por meio dos conselhos de saúde. Ciênc Saúde Colet 2013; 18:7-16.

4. Boivin A, L'Espérance A, Gauvin FP, Dumez V, Macaulay AC, Lehoux P, et al. Patient and public engagement in research and health system decision making: a systematic review of evaluation tools. Health Expect 2018; 21:1075-84.

5. Serapioni M, Duxbury N. Citizens' participation in the Italian health-care system: the experience of the Mixed Advisory Committees. Health Expect 2014; 17:488-99.

6. Serapioni M, Matos AR. Citizen participation and discontent in three Southern European health systems. Soc Sci Med 2014; 123:226-33.

7. Pérez-Cuevas R, Guanais FC, Doubova SV, Pinzón L, Tejerina L, Pinto Masis D, et al. Understanding public perception of the need for major change in Latin American healthcare systems. Health Policy Plan 2017; 32:816-24.

8. Rasanathan K, Bennett S, Atkins V, Beschel R, Carrasquilla G, Charles J, et al. Governing multisectoral action for health in low- and middle-income countries. PLoS Med 2017; 14:e1002285.

9. Serapioni M, Lopes Ferreira P, Antunes P. Participação em saúde: conceitos e conteúdos. Notas Económicas 2014; (40):26-40.

10. Conselho Nacional de Saúde. Resolução no 453, de 10 de maio de 2012. Diário Oficial da União 2012; 6 jun.

11. Serapioni M. Os desafios da participação e da cidadania nos sistemas de saúde. Ciênc Saúde Colet 2014; 19:4829-39.

12. Rifkin SB. Examining the links between community participation and health outcomes: a review of the literature. Health Policy Plan 2014; 29 Suppl 2:98-106.

13. Almeida DCR, Cunha EMS. A análise da deliberação democrática: princípios, conceitos e variáveis relevantes. In: Pereira RRC, organizador. Efetividade das instituições participativas no Brasil: estratégias de avaliação. Brasília: Instituto de Pesquisa Econômica Aplicada; 2011. p. 109-23.

14. Mitton C, Smith N, Peacock S, Evoy B, Abelson J. Public participation in health care priority setting: a scoping review. Health Policy 2009; 91:219-28.

15. Conklin A, Morris Z, Nolte E. What is the evidence base for public involvement in healthcare policy? Results of a systematic scoping review. Health Expect 2015; 18:153-65. 
16. Serapioni M. Participação pública nos sistemas de saúde. Uma introdução. Revista Crítica de Ciências Sociais 2018; (117):91-8.

17. Paiva FS, Stralen CJV, Costa PHA. Participação social e saúde no Brasil: revisão sistemática sobre o tema. Ciênc Saúde Colet 2014; 19:487-98.

18. Bispo Júnior JP, Gerschman S. Legitimidade da representação em instâncias de participação social: o caso do Conselho Estadual de Saúde da Bahia, Brasil. Cad Saúde Pública 2015; 31:183-93.

19. Durán PRF, Gerschman S. Desafios da participação social nos conselhos de saúde. Saúde Soc 2014; 23:884-96.

20. Martins PC, Cotta RMM, Mendes FF, Priore SE, Franceschinni SCC, Cazal MM, et al. De quem é o SUS? Sobre as representações sociais dos usuários do Programa Saúde da Família. Ciênc Saúde Colet 2011; 16:1933-42.

21. Ventura CAA, Miwa MJ, Serapioni M, Jorge MS. Cultura participativa: um processo de construção de cidadania no Brasil. Interface (Botucatu) 2017; 21:907-20.

22. Farias Filho MC, Silva AN, Mathis A. Os limites da ação coletiva nos Conselhos Municipais de Saúde. Ciênc Saúde Colet 2014; 19:1911-9.

23. Rifkin SB, Muller F, Bichmann W. Primary health care: on measuring participation. Soc Sci Med 1988; 26:931-40.

24. Baatiema L, Skovdal M, Rifkin S, Campbell C. Assessing participation in a community-based health planning and services programme in Ghana. BMC Health Serv Res 2013; 13:233.

25. Laverack G. Evaluating community capacity: visual representation and interpretation. Community Dev J 2006; 41:266-76.

26. Draper AK, Hewitt G, Rifkin S. Chasing the dragon: developing indicators for the assessment of community participation in health programmes. Soc Sci Med 2010; 71:1102-9.

27. Carlisle K, Farmer J, Taylor J, Larkins S, Evans R. Evaluating community participation: a comparison of participatory approaches in the planning and implementation of new primary health-care services in northern Australia. Int J Health Plann Manage 2018; 33:704-22.

28. Instituto Brasileiro de Geografia e Estatística. População estimada 2018. Rio de Janeiro: Instituto Brasileiro de Geografia e Estatística; 2010.

29. Avritzer L. A participação social no Nordeste. Belo Horizonte: Editora UFMG; 2007.

30. Gibbs G. Análise de dados qualitativos. Porto Alegre: Artmed; 2009.
31. Moreira MR, Escorel S. Conselhos Municipais de Saúde do Brasil: um debate sobre a democratização da política de saúde nos vinte anos do SUS. Ciênc Saúde Colet 2009; 14:795-806.

32. Fuks M. Participação e influência política no conselho municipal de saúde de Curitiba. Revista de Sociologia e Política 2005; (25):47-61.

33. Vieira M, Calvo MCM. Avaliação das condições de atuação de Conselhos Municipais de Saúde no Estado de Santa Catarina, Brasil. Cad Saúde Pública 2011; 27:2315-26.

34. Oliveira ML, Almeida ES. Controle social e gestão participativa em saúde pública em unidades de saúde do Município de Campo Grande, MS, 1994-2002. Saúde Soc 2009; 18:141-53.

35. Sipioni ME, Silva MZ. Democracia e saúde: a prestação de contas como legitimadora da representação no Conselho Municipal de Saúde de Vitória (ES). Saúde Debate 2015; 39:197-209.

36. Shimizu HE, Pereira MF, Cardoso AJC, Bermudez XPCD. Representações sociais dos conselheiros municipais acerca do controle social em saúde no SUS. Ciênc Saúde Colet 2013; 18:2275-84.

37. Coelho JS. Construindo a participação social no SUS: um constante repensar em busca de equidade e transformação. Saúde Soc 2012; 21:138-51.

38. Avritzer L. A qualidade da democracia e a questão da efetividade da participação: mapeando o debate. In: Pires RRC, organizador. Efetividade das instituições participativas no Brasil: estratégias de avaliação. Brasília: Instituto de Pesquisa Econômica Aplicada; 2011.p. 13-24.

39. Burton P. Conceptual, theoretical and practical issues in measuring the benefits of public participation. Evaluation 2009; 15:263-84.

40. Wendhausen A, Cardoso SM. Processo decisório e conselhos gestores de saúde: aproximações teóricas. Rev Bras Enferm 2007; 60:579-84. 


\section{Abstract}

The article aims to analyze the results of a performance assessment model for health councils. The theoretical and methodological frame of reference was the spider graph method, adapted to the reality of health councils. The assessment matrix considered five dimensions with the greatest influence on participation: autonomy, organization, representativeness, community involvement, and political influence. Based on assessment of the indicators, we estimated the performance value for each dimension and located it on the five-axis graph. The matrix was applied to the Health Council in Vitória da Conquista, Bahia State, Brazil. We used document analysis, observation of meetings, and interviews with 18 council members as the data collection techniques. The results show an advanced level of the council's autonomy with adequate structural conditions, but with limitations in financial independence. The organizational dimension reached the maximum level of performance, with regular meetings, availability of information for council members, and functioning of thematic commissions. Representativeness was the dimension with the worst performance, displayed by the weak relationship between the representatives and the organizations. The community involvement dimension displayed an advanced level with high participation by council and non-council members in the meetings and action with numerous proposals. The political influence dimension showed intermediate performance. We observed greater influence by the social representatives on the decision-making process and low capacity for follow-up on policies. The matrix proved adequate and feasible for performance assessment of health councils.

Social Participation; Health Councils; Health Evaluation; Deliberations; Health Policy

\section{Resumen}

El objetivo de este artículo es presentar y analizar los resultados de un modelo de evaluación de desempeño en consejos de salud. El marco referencial teórico metodológico se fundamentó en el método spidergram, adaptado a la realidad de los consejos de salud. La matriz evaluativa consideró las cinco dimensiones de mayor influencia sobre la participación: autonomía, organización, representatividad, implicación comunitaria e influencia política. A partir de la evaluación de los indicadores, se estimó el valor del desempeño de cada dimensión y se localizó en el gráfico con cinco ejes. La aplicación de la matriz se realizó en el Consejo de Salud de Vitória da Conquista, Bahia, Brasil. Se realizó un análisis de documentación, observación de reuniones y entrevistas con 18 consejeros, así como técnicas de recogida de datos. Los resultados demuestran un nivel avanzado de autonomía del consejo con condiciones estructurales adecuadas, aunque con limitaciones en la independencia financiera. La dimensión organización alcanzó el nivel máximo de desempeño con la realización regular de reuniones, disponibilidad de información para los consejeros y funcionamiento de las comisiones temáticas. La representatividad fue la dimensión de peor desempeño, demostrada por la frágil relación de los representantes con las entidades. La dimensión implicación comunitaria presentó un nivel avanzado con una elevada participación de consejeros y no consejeros en las reuniones y perfil de actuación propositivo. La dimensión influencia política obtuvo un nivel intermedio. Se constató una elevada influencia de los representantes sociales en el proceso deliberativo y una diminuta capacidad de seguimiento de las políticas. La matriz utilizada se mostró adecuada y útil para la evaluación de desempeño en los consejos de salud.

Participación Social; Consejos de Salud; Evaluación en Salud; Deliberaciones; Política de Salud
Recebido em 17/Dez/2018

Versão final reapresentada em 18/Jun/2019

Aprovado em 10/Jul/2019 